\title{
Nasal high flow: physiology, efficacy and safety in the acute care setting, a narrative review
}

This article was published in the following Dove Press journal:

Open Access Emergency Medicine

\author{
Tommaso Mauri ${ }^{1,2}$ \\ Yu-Mei Wang ${ }^{3}$ \\ Francesca Dalla Corte ${ }^{4}$ \\ Nadia Corcione' \\ Elena Spinelli ${ }^{1}$ \\ Antonio Pesenti ${ }^{1,2}$ \\ 'Department of Anesthesia, Critical Care \\ and Emergency, Fondazione IRCCS Ca' \\ Granda Ospedale Maggiore Policlinico, \\ University of Milan, Milan, Italy; \\ ${ }^{2}$ Department of Pathophysiology and \\ Transplantation, University of Milan, \\ Milan, Italy; ${ }^{3}$ Department of Critical Care \\ Medicine, Beijing Tiantan Hospital, \\ Capital Medical University, Beijing, \\ People's Republic of China; ${ }^{4}$ Department \\ of Morphology, Surgery and Experimental \\ Medicine, Azienda Ospedaliera- \\ Universitaria Arcispedale Sant'Anna, \\ University of Ferrara, Ferrara, Italy
}

\begin{abstract}
Nasal high flow (NHF) is a promising novel oxygen delivery device, whose mechanisms of action offer some beneficial effects over conventional oxygen systems. It is considered to have a number of physiological effects: it improves oxygenation, dynamic lung compliance, homogeneity and end expiratory lung volume; it decreases anatomical dead space and generates a positive airway pressure that can reduce respiratory rate, the work of breathing, and enhance patient comfort. NHF has been used as a prophylactic tool or as a treatment device mostly in patients with acute hypoxemic respiratory failure such as preoxygenation before intubation, immunocompromised patients and acute heart failure. Moreover, there is some evidence that NHF could be used during procedural sedation. Finally, NHF was deemed to be effective in chronic obstructive pulmonary disease patients with its positive end expiratory pressure effects and dead-space washout. However, careful monitoring is crucial to maximize NHF settings aimed at maximizing patient comfort while limiting the risk of delayed intubation. The present review presents the most updated evidence for NHF use in the adult acute care setting with the goal of providing clinicians with useful insights on the physiologic effects, main clinical indications, and safety issues of NHF treatment.
\end{abstract}

Keywords: nasal high flow, physiological effects, positive end-expiratory, acute respiratory failure, oxygenation therapy

\section{Introduction}

Nasal high flow (NHF) is a noninvasive respiratory support designed to deliver 30$60 \mathrm{~L} /$ minute of a heated, humidified mixture of air and oxygen through specifically designed nasal prongs. NHF allows modification of two main settings - the percentage of oxygen delivered and the rate of gas flow. NHF can deliver a mix of air and oxygen with an inspired oxygen fraction $\left(\mathrm{FiO}_{2}\right)$ ranging between 0.21 and 1.0 and the flow rate ranges from 30 to $60 \mathrm{~L} /$ minute. Thus, NHF is not necessarily a very high flow of pure oxygen as often wrongly referred to in previous publications. Recently, NHF has gained increasing popularity in treating both type I (hypoxemic) and type II (hypoxemic/hypercapnic) acute respiratory failure (ARF) globally. However, the physiologic effects of NHF on the respiratory system function, and its influence on clinical outcomes are still open research fields, with new studies being published every week or so. The present review presents the most updated evidence for NHF use in the adult acute care setting (ie, from the emergency department to the intensive care unit, ICU) with the goal of providing clinicians with useful insights on the physiologic effects, main clinical indications and safety issues of NHF treatment.
Correspondence: Antonio Pesent Department of Anesthesia, Critical Care and Emergency, Fondazione IRCCS Ca' Granda Ospedale Maggiore Policlinico, Via F. Sforza 35, 20122 Milan, Italy

Tel +39025 5033232

Fax +39025 5033230

Email antonio.pesenti@unimi.it 


\section{Physiological effects}

The main physiological effects of NHF are maintenance of a known stable alveolar $\mathrm{FiO}_{2}$, optimal humidification of the airway mucosa, reduction of the dead space, generation of a positive end-expiratory pressure (PEEP), ultimately yielding a reduction in the respiratory drive and in the work of breathing (see Table 1).

\section{Stable alveolar $\mathrm{FiO}_{2}$}

Low-flow nasal cannulas are connected to the hospital gas delivery system via flowmeters, most of which allow delivery of gas flows up to $15 \mathrm{~L} /$ minute. Traditional nasal cannula flow rate has been limited, not only due to the internal diameter of the cannula, but also by the risks associated with the lack of heating and humidification of the inspired gas. In dyspneic ARF patients, when the inspiratory flow rate exceeds the flow delivered, additional flow is entrained from the surrounding room air. Consequently, traditional low flow nasal cannula cannot deliver constant alveolar $\mathrm{FiO}_{2}$, even at the highest flow rates. ${ }^{1,2}$ NHF very high flow rates can match the inspiratory demand of dyspneic patients and overcome the need of additional room air-mixing, finally providing stable delivered alveolar $\mathrm{FiO}_{2}{ }^{3}$ In the abovementioned range from 30 to $60 \mathrm{~L} /$ minute, bench studies showed that the $\mathrm{FiO}_{2}$ measured in distal airways is very close to the set $\mathrm{FiO}_{2}{ }^{4}$ Recently, Parke et $\mathrm{al}^{5}$ performed a study on healthy volunteers with NHF delivered with flow exceeding $60 \mathrm{~L} /$ minute. If heating and humidification can be granted as such high flow rates, this study suggests that alveolar $\mathrm{FiO}_{2}$ during NHF could be high and stable even in the most severe patients. NHF, in the presence of stable oxygen consumption and $\mathrm{CO} 2$ production, likely improves the correspondence between set and alveolar $\mathrm{FiO} 2$, but the extent of this benefit is difficult to predict in clinical practice.

\section{Humidification}

Conventional oxygen devices delivering dry and cold gases usually causes discomfort, nasal and oral dryness,

Table I Key physiological benefits of NHF, divided between those already described in recent literature and still to be assessed in prospective studies

\begin{tabular}{|c|c|}
\hline \multicolumn{2}{|l|}{ Key physiological benefits of NHF } \\
\hline \multicolumn{2}{|l|}{ Already described } \\
\hline Improvement of oxygenation & $\begin{array}{l}\text { Mauri et al , Am J Respir Crit Care Med } 2017^{20} \\
\text { Möller et al, J Appl Phyiol } 2017^{11}\end{array}$ \\
\hline Reduction of respiratory rate & $\begin{array}{l}\text { Mauri et al, Am J Respir Crit Care Med } 2017^{20} \\
\text { Corley et al, Br J Anaesth 201 }{ }^{15}\end{array}$ \\
\hline Increased $\mathrm{CO}_{2}$ clearance & $\begin{array}{l}\text { Möller et al, J Appl Physiol } 2017^{11} \\
\text { Mauri et al, Am J Respir Crit Care Med } 2017^{20}\end{array}$ \\
\hline PEEP effect (increased pharyngeal pressure and lung volume) & $\begin{array}{l}\text { Parke et al, Respir Care } 20 \mathrm{II}^{28} \\
\text { Corley et al, Br J Anaesth } 20 \mathrm{II}^{15}\end{array}$ \\
\hline Reduction of work of breathing & $\begin{array}{l}\text { Sztrymf et al, Intensive Care Med } 201 \mathrm{I}^{22} \\
\text { Itagaki et al, Respir Care } 2014^{23} \\
\text { Mauri et al, Intensive Care Med } 2017^{25}\end{array}$ \\
\hline Limit the risk of P-SILI: decrease lung stress, strain, heterogeneity & Mauri et al, Am J Respir Crit Care Med $2017^{20}$ \\
\hline \multirow[t]{3}{*}{ Optimal comfort } & Roca et al, Respir Care $2010^{26}$ \\
\hline & Mauri et al, Critical Care $2018^{76}$ \\
\hline & Lenglet et al, Respir Care $2012^{64}$ \\
\hline \multicolumn{2}{|l|}{ Still hypothetical } \\
\hline \multicolumn{2}{|l|}{ Improved matching between set and alveolar $\mathrm{FiO}_{2}$} \\
\hline \multicolumn{2}{|c|}{ Optimization of mucociliary cells function } \\
\hline \multicolumn{2}{|c|}{ Reduced $\mathrm{CO}_{2}$ production from mucosal epithelium and inspiratory muscles } \\
\hline \multicolumn{2}{|c|}{ Decreased respiratory drive } \\
\hline \multicolumn{2}{|l|}{ Increased expiratory transpulmonary pressure } \\
\hline Decreased inspiratory transalveolar pressure & \\
\hline
\end{tabular}

Abbreviations: NHF, nasal high flow; $\mathrm{CO}_{2}$, carbon dioxide; PEEP, positive end-expiratory pressure; P-SILI, patients' self-inflicted lung injury; FiO ${ }_{2}$, fraction of inspired oxygen. 
eye irritation, nasal and eye trauma. ${ }^{6,7}$ Inspired gases, when unwarmed and dry, may yield a variety of untoward effects on ARF patients on respiratory support. As the airway dries and cool down, mucociliary function gets impaired and it is more difficult to clear the airway of secretions. NHF, instead, undergoes $100 \%$ humidification and is heated up to approximately normal body temperature. Effective humidification and heating will cause less mucociliary dessication, ${ }^{8}$ thus granting more efficient mucociliary clearance, facilitating clearance of secretions, and decreasing the risk of occlusion atelectasis, finally resulting in improved ventilation/perfusion matching and oxygenation. Better humidification and heating could mean improved comfort, too. Saslow et al described greater compliance in infants supported with $5 \mathrm{~L}$ /minute of NHF with conditioned gas compared to $6 \mathrm{~cm} \mathrm{H}_{2} \mathrm{O}$ of conventional continuous positive airway pressure (CPAP). ${ }^{9}$

\section{Dead space washout}

The continuous high flow rate provided by NHF washes the residual volume of carbon dioxide $\left(\mathrm{CO}_{2}\right)$ lying in the upper airways from previous breath and replaces it with $\mathrm{CO}_{2}$-free oxygen-enriched gas. In a lung-injured-animal model, $\mathrm{PaCO}_{2}$ decreased as NHF flow increased, as higher rates of gas flow more effectively washed out $\mathrm{CO}_{2}$. These results suggest effective carbon dioxide washout with NHF. ${ }^{10}$ Moreover, in healthy volunteers, Möller et al showed rapid and effective wash out of radiological tracer from the upper airways by NHF. ${ }^{11}$ As patients using the NHF may open or close their mouth at will, this might influence alveolar $\mathrm{FiO}_{2}$ and $\mathrm{CO}_{2}$ washout. Wettstein et al compared $\mathrm{FiO}_{2}$ in healthy volunteers breathing with mouth open and closed ${ }^{12}$ and, counterintuitively, $\mathrm{FiO}_{2}$ was higher during mouth-open breathing. This may have been due to the reservoir function of the nose, the pharynx, and, potentially, the oral cavity after being washed out from exhaled $\mathrm{CO}_{2}$. By allowing NHF to continually suffuse the nasal cavity during exhalation, breathing with the mouth open may enable more efficient $\mathrm{CO}_{2}$ washout and provide a larger anatomic reservoir. Thus, these mechanisms suggest that $\mathrm{CO}_{2}$ washout may contribute to the observed increase in alveolar $\mathrm{FiO}_{2}$.

\section{PEEP effect and lung volumes}

Research measuring pressure in the lower pharynx showed positive linear correlation between PEEP and the amount of flow delivered by NHF. Moreover,
Groves et $\mathrm{al}^{13}$ described that PEEP is determined by flow also in the presence of mouth open. In healthy volunteers, mean airway pressure associated with NHF at $0,1020,40$ and $60 \mathrm{~L} /$ minute with the mouth closed was $0.8,1.7,2.9,5.5$ and $7.7 \mathrm{~cm} \mathrm{H}_{2} \mathrm{O}$, respectively. Similar positive pressure effect was reported in the studies performed by Ritchie et $\mathrm{al}^{14}$ and Corley et $\mathrm{al}^{15}$ in hypoxemic patients. Moreover, Parke et $\mathrm{al}^{5}$ performed a study on healthy volunteers to describe if the correlation remains linear at flows exceeding $50 \mathrm{~L} / \mathrm{min}$ ute. On average, for every $10 \mathrm{~L} /$ minute increase in gas flow, the generated mean airway pressure increased by $1.16 \mathrm{~cm} \mathrm{H}_{2} \mathrm{O}$. NHF support was associated with an increase in end-expiratory lung volume (EELV), which indicates positive alveolar pressure at end-expiration, a decrease in respiratory rate (RR), and a more uniform distribution of alveolar ventilation between lung regions. The increase of EELV related to the use of NHF seems independent from changes in body position. Riera et $\mathrm{al}^{16}$ described an increase in EELV with the use of NHF in both supine and prone positions. Plotnikow et $\mathrm{al}^{17}$ assessed EELV changes induced by NHF use at different flows in healthy subjects in the semi-seated position. Global and regional EELV measured by electrical impedance tomography also increased with increasing flows. A strong correlation between airway pressure and increase of EELV was described by Corley et $\mathrm{al}^{15}$ in adult patients treated with NHF after cardiothoracic surgery. Compared with conventional oxygen therapy, NHF increased EELV, reduced the respiratory rate and increased the tidal volume (Vt). Finally, in the research performed by Mündel et $\mathrm{al}^{18}$ who used an elastic sensor belt for polysomnography to measure Vt in healthy volunteers, NHF led to a marked increase in Vt. Okuda et $\mathrm{al}^{19}$ also demonstrated Vt increase at different flows and Vt seemed to correlate with NHF flow rate. Corley et $\mathrm{al}^{15}$ reported similar findings, too. However, Mauri et $\mathrm{al}^{20}$ described stable Vt in hypoxemic patients during NHF vs low-flow oxygen mask and Bräunlich et $\mathrm{al}^{21}$ reported decreased $\mathrm{Vt}$ in healthy volunteers during NHF. It might be possible that patients with acute lung injury supported by NHF do not reduce the tidal volume because of disease-related high respiratory drive. Thus, conclusions on the effects of NHF on $\mathrm{Vt}$ are still lacking, and differences in age, sex, clinical condition and the methods used to measure Vt may explain heterogeneous findings. 


\section{Reduction of respiratory drive}

Nearly all studies conducted both in healthy volunteers and in ARF patients reported decreased respiratory rate and improved dyspnea during NHF compared to standard low-flow oxygen. These findings suggest that the central respiratory drive could be reduced by NHF, likely through the interaction between all the abovementioned mechanisms: increased alveolar $\mathrm{FiO}_{2}$ could lead to improved oxygenation and decreased hypoxic drive, effective humidification could reduce discomfort, dead space washout reduces the minute ventilation needed to maintain stable arterial $\mathrm{CO}_{2}$ level and the hypercapnic drive, and finally the PEEP effect could contribute to improved oxygenation and to improved respiratory system mechanics with easier ability to inspire the desired $\mathrm{Vt}^{20}$

\section{Reduction of work of breathing}

NHF could also decrease the work of breathing, depending on the clinical condition of the patient. Sztrymf et $\mathrm{al}^{22}$ showed an improvement in subjective measures of inspiratory load by NHF in the patients diagnosed as having poor thoraco-abdominal coordination under standard low-flow oxygen. The same results were obtained by Itagaki et $\mathrm{al}^{23}$ in patients with mild to moderate respiratory failure treated with the NHF after thoracotomy. Previous studies also described the reduction of inspiratory effort and work of breathing by NHF, as quantified by esophageal pressure inspiratory swings in pediatric populations. ${ }^{9,24}$ Mauri et $\mathrm{al}^{25}$ performed a prospective randomized crossover study in adults nonintubated patients with hypoxemic ARF. Measures of inspiratory effort and metabolic work of breathing assessed by esophageal pressure significantly decreased during NHF therapy (Figure 1). The authors also suggested that NHF could improve other key physiologic parameters including dynamic lung compliance, transpulmonary pressure, and homogeneity. Similar effects on reduction of effort and work of breathing were reported in stable patients with COPD (see the section below).

\section{Clinical indications in hypoxemic patients}

\section{Acute hypoxemic respiratory failure}

NHF is becoming the first-line treatment for acute hypoxemic respiratory failure (AHRF) patients when standard oxygenation via facial mask or nasal cannula yields poor oxygenation and immediate intubation is not required.
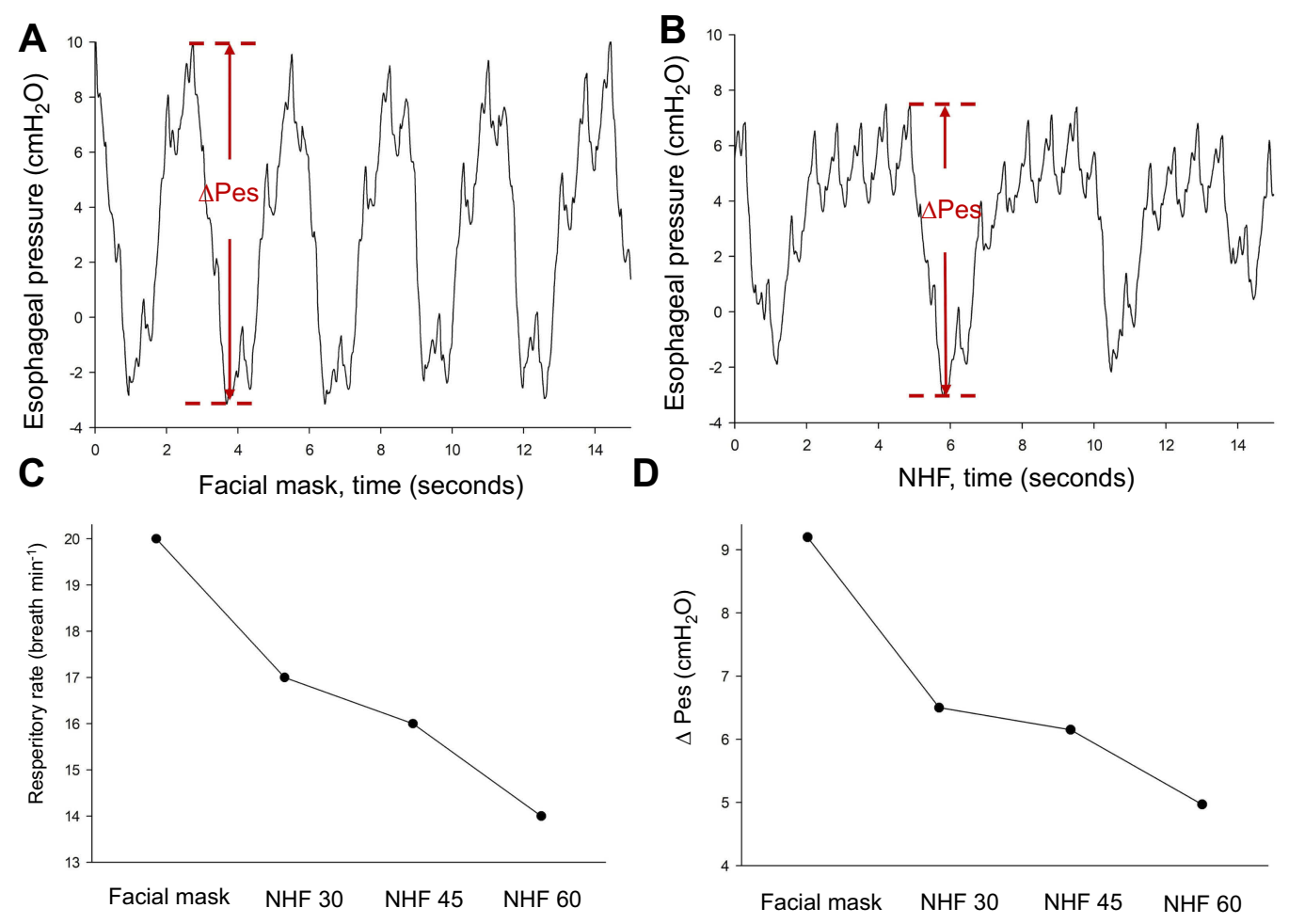

Figure I Tracing of esophageal pressure swings ( $\Delta$ Pes) during $(\mathbf{A})$ low flow oxygenation compared to (B) nasal high flow support, showing reduced inspiratory effort. Indeed, respiratory rate $(\mathbf{C})$ and inspiratory effort $(\mathbf{D})$ decreased with increasing NHF flow rate.

Abbreviations: NHF, nasal high flow; $\triangle$ Pes, esophageal pressure swings. 
The benefits of NHF therapy compared to standard oxygen has been widely described since the first study of Roca et $\mathrm{al}^{26}$ in adult patients in 2010. It reported an increase in both $\mathrm{SaO}_{2}$ and $\mathrm{PaO}_{2}$ and comfort with a decrease of respiratory rate and dyspnea. These results were further confirmed by Sztrymf et $\mathrm{al}^{27}$ who also reported important hints on early recognition of NHF failure and the need for consideration for intubation. ${ }^{22}$ Compared to conventional oxygen therapy, NHF could be more effective in mild to moderate AHRF also in reducing the need for escalation to noninvasive ventilation $(\mathrm{NIV}) .^{28}$

But it was not until the first large randomized clinical trial by Frat et al, ${ }^{29}$ the FLORALI trial, that NHF administered at $50 \mathrm{~L} /$ minute was compared both to NIV (for at least 8 hours per day) and standard oxygen (face mask at $10 \mathrm{~L} /$ minute or more) in terms of hard clinical outcomes. In patients with a $\mathrm{PaO}_{2} / \mathrm{FiO}_{2}$ ratio $\leq 300$ with AHRF $(84 \%$ with pneumonia as primary diagnosis), NHF was not superior to the other two treatments in lowering intubation rate, the primary outcome. However, a post-hoc analysis found that NHF significantly reduced intubation in the subgroup with $\mathrm{PaO}_{2} / \mathrm{FiO}_{2}$ ratio $\leq 200$. Ventilatorfree days and 90 day mortality, described as secondary outcomes, were reduced by NHF in the whole population, too.

These results were challenged by a second large randomized clinical trial, the HOT-ER trial, ${ }^{30}$ comparing the administration of NHF starting from $30 \mathrm{~L} /$ minute to face mask oxygenation up to $15 \mathrm{~L} /$ minute in the emergency department. NHF resulted in lower rates of intubation after 24 hours (although with a marginal statistical significant, $P=0.053$ ). No differences in mortality at 90 days was found. However, HOT-ER population was different and very heterogeneous, with lower percentage of community acquired pneumonia patients and without the exclusion of COPDand asthma exacerbations.

Two recent systematic reviews and meta-analyses were published to better clarify these aspects. The first included over 3,000 AHRF patients and described that NHF reduced the need for endotracheal intubation compared to conventional oxygen and NIV (OR: 0.60, 95\%CI:0.41-0.86). ${ }^{31}$ The second ${ }^{32}$ included 1,084 patients and reported that, compared to conventional oxygen and NIV, NHF could reduce both the rate of endotracheal intubation (OR: 0.62 and OR: 0.48, respectively) and ICU mortality (OR: 0.47 and OR:
0.36, respectively) when used before mechanical ventilation.

Although still relatively few, these data seem to indicate that NHF should be considered as first-line therapy for patients with AHRF.

\section{Acute hypoxemic respiratory failure in immunosuppressed patients}

Intubation in immunosuppressed patients with AHRF is associated with extremely elevated mortality, hence a respiratory management aiming to avoid intubation should be promoted.

A post-hoc analysis of the FLORALI trial showed that NIV increased intubation and mortality compared to NHF or conventional oxygen in immunosuppressed patients. ${ }^{33}$ The same results were obtained in a prospective observational study with NHF compared to NIV, both used as firstline therapy. ${ }^{34}$ Moreover, NHF compared to NIV and face mask oxygen, respectively, was associated with lower mortality in specific cohorts, such as cancer patients ${ }^{35}$ and lung transplantrecipients. ${ }^{36}$

More recently, the HIGH randomized clinical trial ${ }^{37}$ compared continuous NHF therapy to standard oxygen therapy in 778 immunocompromised patients with AHRF. Azoulay et al found no statistically significant differences in 28-day mortality $(35.6 \%$ vs $36.1 \%$, $P=0.94)$, intubation rate $(38.7 \%$ vs $43.8 \%, P=0.17)$ and ICU mortality ( $31.7 \%$ vs $31.4 \%, P=0.77$ ), with no comfort and dyspnea score improvement by NHF therapy. However, the population was very heterogeneous and the authors concluded that attention to oxygenation strategies may not be the main focus in such a diverse population.

\section{Preoxygenation for intubation}

The main goal of preoxygenation is to extend the period of safe apnea, thus avoiding a desaturation below $88 \%$ during intubation manuever. This is usually achieved by obtaining an arterial oxyhemoglobin saturation as close as possible to $100 \%$ and by the denitrogenation of the lungs (ie, the wash out of nitrogen contained in room air, increasing alveolar oxygen reservoir). These aspects might be both promoted by the dead-space washout and PEEP effect of the NHF therapy. Moreover, compared to other facial devices, NHF support can be kept during the maneuver.

In the operating theatre, preoxygenation with NHF was applied to a cohort of 50 patients undergoing scheduled awake fiberoptic intubation for expected difficult airways 
management. The therapy was generally well tolerated, with no desaturation below $90 \%$ despite the length of procedures (up to 17 minutes). ${ }^{38}$ In the same clinical setting, NHF at $70 \mathrm{~L} /$ minute for 10 minutes during induction was tested to deliver apneic oxygenation: Patel et $\mathrm{al}^{39}$ associated NHF oxygenation to jaw thrust to increase apnea time during induction of general anesthesia and difficult airways management with no desaturation and stable $\mathrm{CO}_{2}$ level. These results were further confirmed by a randomized clinical trial, highlighting NHF potential compared to face mask during preoxygenation for rapid sequence induction of anesthesia. ${ }^{40}$

In critically ill patients, given the possible combination of reduced pulmonary functional residual capacity and a compromised cardiovascular reserve, achieving an optimal oxygenation status during intubation is mandatory. Despite preliminary promising evidences, ${ }^{41}$ preoxygenation by NHF did not prevent occurrence of severe hypoxemia compared to standard clinical practice (ie administering oxygen through a non-rebreathing bag reservoir facemask) ${ }^{42}$ irrespective of baseline patients' oxygenation status. ${ }^{43}$ It could be inferred that in patients with AHRF as primary reason for intubation, the PEEP and the apneic oxygenation effects induced by NHF might not avoid desaturation. Conversely, NIV effectiveness has been proved for preoxygenation in patients with severe AHRF. $^{44}$ Moreover, the combination of NHF with NIV seems to maintain higher $\mathrm{SpO}_{2}$ levels during induction with no severe desaturation $\left(\mathrm{SpO}_{2}<80 \%\right)$ compared to NIV alone, since NHF and apneic oxygenation can be continued after NIV is removed. ${ }^{45}$ The final word on NHF vs NIV for preoxygenation in AHRF critically ill patients might come from ongoing trials. ${ }^{46}$

\section{Acute heart failure}

Through the generation of low levels of PEEP and improved oxygenation, HFNC resulted in positive hemodynamic changes in New York Heart Association class III patients as indicated by significantly decreased inspiratory collapse of the inferior vena cava and thus the preload of the right ventricle. ${ }^{47}$ Consequently, NHF therapy seems promising in acute cardiogenic pulmonary edema. However, limited evidence exists on its application in this context: Carratala Perales et $\mathrm{al}^{48}$ showed significant improvement of the intensity of dyspnea, respiratory rate and oxygenation after 24 hours of HFNC treatment in 5 patients with acute heart failure and refractory hypoxemia despite NIV. To date, no randomized clinical trial has been published comparing NHF with NIV or standard oxygen in acute heart failure or acute myocardial infarction patients.

\section{Procedural sedation}

Procedural sedation is widely used to achieve safe adequate patient tolerance of several invasive procedures in the respiratory medicine, interventional gastroenterology, and cardiology fields. Due to the expected side effects of the sedative drugs (eg, propofol, benzodiazepines, opioids), the respiratory drive is depressed, reflexes are inhibited and upper airways might be occluded. Hence, oxygen saturation may fall despite oxygen supplementation.

NHF has been proposed as an ideal oxygenation device since it allows oral passage of the operative tools while improving oxygenation during the procedure through PEEP effect and additional oxygen. Lucangelo et al prospectively randomized 45 nonhypoxemic patients undergoing bronchoscopy to NHF set at $40 \mathrm{~L} /$ minute with $50 \% \mathrm{FiO}_{2}$, NHF set at $60 \mathrm{~L} /$ minute with $50 \% \mathrm{FiO}_{2}$, or Venturi mask with $50 \% \mathrm{FiO}_{2}$ during the procedure. ${ }^{49}$ With similar sedative drugs dosage and procedure duration among groups, NHF set at $60 \mathrm{~L} /$ minute granted more adequate oxygenation (higher $\mathrm{SpO}_{2}$ and $\mathrm{PaO}_{2} / \mathrm{FiO}_{2}$ compared to the other treatments) at the end and shortly after the procedure, thus being a reasonable option during routine bronchoscopy in patients with mild respiratory dysfunction.

Simon et $\mathrm{al}^{50}$ compared the use of NHF at $50 \mathrm{~L} / \mathrm{min}$ to NIV in critically ill patients with moderate to severe hypoxemia during bronchoscopy: patients treated with NIV showed better oxygenation during and after the procedure, however patients treated by NHF maintained acceptable peripheral oxygenation throughout the procedure (minimum value $92 \pm 7 \%$ ). In a similar cohort of patients, La Combe et $a l^{51}$ tested the efficacy of NHF therapy at $50-60 \mathrm{~L} / \mathrm{min}$ with only $5 / 30$ patients requiring escalation of therapy within $24 \mathrm{hrs}$ after bronchoscopy. These studies suggest the efficacy and safety of NHF during bronchoscopy also in patients with compromised respiratory function.

NHF therapy seems promising as respiratory support also in procedural sedation during dental surgery, ${ }^{52}$ and during percutaneous balloon aortic valvuloplasty under continuous monitoring with transesophageal echocardiography. ${ }^{53}$ Finally, in a retrospective analysis of 238 patients undergoing endoscopic retrograde cholangiopancreatography, ${ }^{54} \mathrm{NHF}$ use during deep sedation might be useful to avoid general anesthesia in those patients considered at high risk of perioperative mortality. 


\section{Clinical indications for COPD patients}

According to the World Health Organization, 65 million people worldwide suffer from moderate to severe COPD, which is supposed to become the third leading cause of death by $2020 .{ }^{55,56}$ NIV is the first-line respiratory support for hypercapnic ARF. However, it is applied intermittently, usually with periods of unsupported spontaneous breathing with conventional $\mathrm{O}_{2}$ interspersed between NIV sessions. Standard $\mathrm{O}_{2}$ has several drawbacks that may limit the benefit of intermittent NIV in hypercapnic ARF: limited gas flow; cold and dry gases leading to discomfort and under-humidification. In this scenario, the role of NHF could open new perspectives in the management of both acute and chronic hypercapnic COPD patients, when applied during breaks from NIV, and a dedicated study might unveil the advantages of NHF support soon. ${ }^{57}$

During COPD both lung compliance and airway resistance increase and the physiologic breathing pattern with lower respiratory rate and higher variability could be beneficial in COPD patients. In COPD patients, the PEEP generated by NHF could stent the early-collapsing bronchioles, counterbalancing-at least in part-the intrinsic positive end-expiratory pressure (PEEPi), and improving the alveolar gas exchange (both hypoxia and hypercapnia). As the end-expiratory airway pressure is increased by NHF, the work of breathing needed to overcome both elastic and resistive components is lower for the same tidal volume. Being the initial airway pressure higher with the small PEEP generated by NHF, the work of breathing, which should overcome both elastic and resistive components of the lung, is lower for the same tidal volume during NHF . More than the PEEP effect, two major mechanisms of NHF can provide a strong pathophysiological rationale for its application in hypercapnic COPD patients: (1) the reduction of work of breathing via the reduction of inspiratory resistance: as the upper airway resistance from nares to trachea constitutes about two thirds of total airway resistance, NHF reaches past the nasal valve leading to a significant reduction of inspiratory work of breathing. As is well documented in an elegant study by Adams et al, ${ }^{58}$ during the inspiratory phase, NHF rates elevate tracheal pressure, but the latter reaches more rapidly the zero and negative pressure; then, from this point and until the end of inspiration, no muscular efforts are spent to overcome the upper airway resistance; (2) the washout of upper airways dead space. Recently, Biselli et al have reported that in COPD patients during sleep, the degree of physiological dead space at baseline correlates with its reduction during NHF. ${ }^{59}$ Interestingly, the reduction in minute ventilation observed during NHF was due to a reduction of $\mathrm{Vt}$ without changes in $\mathrm{RR}$ apart from one case. It is important to underline that despite the decrease of $\mathrm{Vt}$, NHF was associated to a significant reduction in transcutaneous $\mathrm{CO}_{2}$, indicating higher efficiency of the minute ventilation.

\section{Acute hypercapnic respiratory failure}

Kim et al performed a single center retrospective study on thirty-tree patients admitted to the medical intensive care unit for ARF with hypercapnia $\left(\mathrm{PaCO}_{2} 56.3 \pm 10.7 \mathrm{mmHg}\right)$ and treated with conventional oxygen therapy (COT) or NIV. ${ }^{60}$ NHF was applied when the patients did not improve or for intolerance to NIV. Once started, NHF decreased $\mathrm{PaCO}_{2}$ after both 1 and 24 hours of treatment, resulting in a significant improvement of hypercapnia; furthermore, NHF efficacy was irrespective of whether chronic hypercapnia was present or not. However, a similar decrease in $\mathrm{PaCO}_{2}$ was obtained with COT and NIV, too. It should be noted that patients in this study might have already achieved a clinical stability when started on NHF, as indicated by the absence of respiratory acidosis despite hypercapnia (mean $\mathrm{PaCO}_{2} \mathrm{mmHg}$, but mean normal $\mathrm{pH}$ was 7.37). Thus, we cannot exclude that the initial treatment with NIV or simply the medical therapy was the determinant of hypercapnia correction, independently from the type of respiratory support. Furthermore, NHF was applied if no clinical improvement with COT or NIV was observed, but "no clinical improvement" does not necessarily mean "clinical failure". Finally, the etiology of respiratory failure was extremely heterogeneous (pneumonia, acute exacerbation of COPD, worsening of interstitial lung disease, cardiogenic edema, extrapulmonary acute lung injury) and no clinical severity score was reported. The study by Kim et alshowed feasibility of NHF as an alternative to COT and NIV in stable COPD patients.

In another prospective observational trial, ${ }^{61}$ Lee et alcompared the effectiveness of NHF and NIV for the treatment of severe acute exacerbation of COPD (AECOPD) with moderate hypercapnia. The primary endpoint was the intubation rate and 30-day mortality. The 
patients were randomly assigned to receive either NIV or NHF. The authors reported that $\mathrm{PaO}_{2}, \mathrm{PaCO}_{2}$ and $\mathrm{pH}$ collected at 6 and 24 hours from the beginning of the treatment were not significantly different between groups. No difference in intubation rate nor in 30-day mortality was found. Notably, among the 88patients studied, the etiology of AECOPD was unknown in 18; in the remaining 70 patients, the etiology was pneumonia in 37 patients and upper respiratory tract infection in the other 21 patients. Similarly to the study by Kim et al, grouping under the definition of AECOPD different pathologies makes it difficult to interpret the net effect of NHF.

The clinical efficacy and safety of NHF compared to low-flow oxygen via nasal cannulae or mask, continuous positive airway pressure and bi-level positive airway pressure, in adult COPD patients hospitalized in the ICU, were analysed in the 2017 Cochrane systematic review. Pooled data analysis revealed no differences in carbon dioxide clearance between groups. ${ }^{62}$

Finally, in a large multicenter randomized trial on adults presenting to the emergency department with mixed hypoxemic or hypercapnic respiratory failure requiring noninvasive ventilation, ${ }^{63}$ patients were randomly assigned to receive NHF or NIV. The study evidenced similar improvement in the $\mathrm{PaCO}_{2}$ levels over time in both arms, in the whole population and in the subgroup with initial $\mathrm{PaCO}_{2}$ greater than $45 \mathrm{mmHg}$.

These studies indicate that NHF is feasible and safe in the treatment of AECOPD patients and might be correlated with improved physiology, like NIV but with a much easier setup. Well conducted randomized trials in AECOPD comparing the clinical efficacy of HFNC vs COT or NIV are still lacking. The etiology of hypercapnia should become a more central focus to move forward with future studies.

\section{NHF in the emergency department}

The use of NHF has been reported in the whole spectrum of the critical care settings, including ICUs and perioperative medicine, both for hypoxic and hypercapnic ARF. There issome initial evidence on the use of NHF in patients admitted to the emergency department (ED) with dyspnea, too. A prospective, observational study was conducted in the ED by Lenglet et al. ${ }^{64}$ Their study showed physiological improvements of dyspneic patients by NHF, such as decreased RR, and dyspnea scores and increased $\mathrm{SpO}_{2}$. Bell et al ${ }^{65}$ enrolled 100 patients presenting at the ED with shortness of breath in a randomized controlled trial comparing NHF with conventional $\mathrm{O}_{2}$. They described that the use of NHF was associated with greater reduction in RR $(>20 \%$ from baseline) and lower need for escalation of respiratory support. These results suggest that use of NHF is feasible and effective in the ED and could be considered first-line support in patients with acute shortness of breath. Makdee et al ${ }^{66}$ also found that NHF may decrease the severity of dyspnea during the first hour of treatment in ARF due to congestive heart failure in the ED, compared to COT.

Since these data are not sufficient to define feasibility and efficacy of NHF in patients presenting with ARF at the $\mathrm{ED}$, a few concerns should be brought to the attention of emergency physicians. ${ }^{67}$ When a patient is admitted to the $\mathrm{ED}$, the primary aim of the attending physician should be to perform accurate diagnosis. Before starting NHF, several aspects of the patient's condition should be evaluated, including severity of illness and need for ICU admission. Indeed, NHF can mask severe conditions, delaying diagnosis and escalation of treatment. Initial NHF settings could be based on diagnosis, too, as more hypoxic patients or those with acute cardiogenic pulmonary edema could benefit more from higher flow rate, while a lower rate could be selected in hypercapnic ones. During NHF support, it is important to frequently reevaluate respiratory parameters, including $\mathrm{RR}, \mathrm{SpO}_{2}$, dyspnea score and comfort to detect early NHF failure. ${ }^{68}$

\section{Safety issues during NHF support}

One of the main concern during NHF support is the risk of delayed endotracheal intubation in hypoxemic patients. In a retrospective observational study, Kang et $\mathrm{al}^{69}$ showed how NHF might unduly delay initiation of mechanical ventilation and worsen patient outcome. They reported that patients intubated after 48 hours from HFNC initiation had higher overall ICU mortality after propensity score adjustment and matching vs patients intubated within 48 hours. However, the criteria for intubation were not prospectively standardized and the patients with delayed intubation might have simply been overlooked by the staff physicians. ${ }^{70}$ Moreover, in the Kang et al study, median duration of NHF support in the group intubated after more than 48 hours was 126 hours in comparison with 10 hours in the $<48$ hours group. This induced Ricard et al ${ }^{71}$ to ask whether leaving patients in respiratory distress for more than 5 days, with all the associated clinical implications, could have had a major impact on ICU mortality. Still, failure to improve within 48 hours should be seen as a sign of more severe lung injury. To this end, Roca et $\mathrm{al}^{72,73}$ proposed a new index, the ROX index, calculated as 
$\left(\mathrm{SpO}_{2} / \mathrm{FiO}_{2}\right) / \mathrm{RR}$, to identify at 12 hours the patients at higher risk of NHF failure. A ROX index $\geq 4.88$ points can identify patients who will likely heal, while ROX $<3.85$ is associated with high probability of ending up intubated. Other signs of respiratory distress such as respiratory rate, use of accessory respiratory muscles, presence of thoraco-abdominal asynchrony and discomfort measured within the first hour ofNHF initiation were associated with high risk of failure. ${ }^{27,74}$ Together with the abovementioned clinical parameters, the radiological analysis of chest X-ray has been considered to predict failure. Koga et $\mathrm{al}^{75}$ described that the extent of pleural effusion at NHF initiation was associated with failure. Lung imaging (ie chest X-ray, lung ultrasound or EIT) could have an important role in the early identification of patients with extensive opacities or effusions and eventually failing NHF.

Major safety issues associated with use of NHF in the neonatal population, such as pneumothorax or burn lesions, are not reported in adults. However, another drawback might be severe patient discomfort. In a recent posthoc analysis of the FLORALI trial, Frat et $\mathrm{al}^{74}$ reported higher patient discomfort after 1 hour in patients failing NHF support vs those who were not intubated.

Patient discomfort might be also linked to insufficient air humidification/high temperature. Mauri et al ${ }^{76}$ reported that, independently from the set flow rate, lower temperature with full humidification is associated with lower discomfort.

In a study performed in the $\mathrm{ED},{ }^{64}$ both patients $(100 \%)$ and caregivers $(82 \%)$ judged NHF to be more comfortable compared to standard oxygenation therapy. In the same study, noise level generated by NHF ( $55 \mathrm{~dB}$ ) was comparable to standard oxygen face mask $(50 \mathrm{~dB})$ and not higher than ambient noise $(60-70 \mathrm{~dB})$. Altogether, $76 \%$ of caregivers preferred NHF, compared to COT.

Available data in recent literature indicates that, despite the significant advantages provided by NHF to the management of ARF patients, around $30 \%$ of them will fail and require invasive ventilation. Hence, ED physicians should keep in mind that patients presenting higher complexity should be monitored closely, for example presence of an additional extrapulmonary organ failure is an early indicator of risk of failure. ${ }^{77}$ In another study, Messika et $\mathrm{al}^{78}$ reported that in a cohort of ARF patients the Simplified Acute Physiology Score II was higher in patients failing NHF, mostly due to additional hemodynamic and neurologic dysfunctions. Roca et $\mathrm{al},{ }^{36}$ in a cohort of lung transplant recipients re-admitted to the ICU and supported by NHF, found that patients with bilateral infiltrates and those needing vasopressors during their ICU stay were more likely to end up intubated. Consistent with these results was the finding by Koga et $\mathrm{al}^{75}$ of a higher sequential organ failure assessment score in ARF patients failing NHF, with similar relevance for all the components of the score.

\section{Conclusion}

NHF is a powerful noninvasive respiratory support that can positively impact the physiology of acute and chronic respiratory failure patients. However, studies showing translational benefits on hard clinical outcomes are still to come in most patient populations. Careful monitoring is crucial to maximize NHF benefits while limiting the risk of delayed intubation.

\section{Disclosure}

Dr T Mauri received speaking fees for lectures during symposia by Fisher and Paykel outside the submitted work. The authors report no other conflicts of interest in this work.

\section{References}

1. Ward JJ. High-flow oxygen administration by nasal cannula for adult and perinatal patients. Respir Care. 2013;58:98-122. doi:10.4187/ respcare.01941

2. Nishimura M. High-flow nasal cannula oxygen therapy in adults. $J$ Intensive Care. 2016;3:15. doi:10.1186/s40560-015-0084-5

3. Helviz Y, Einav S, Systematic A. Review of the high-flow nasal cannula for adult patients. Crit Care. 2018;22(1):71. doi:10.1186/ s13054-017-1926-4

4. Nishimura M. High-flow nasal cannula oxygen therapy in adults. $J$ Intensive Care. 2015;3(1):15. doi:10.1186/s40560-015-0084-5

5. Parke RL, Bloch A, McGuinness SP. Effect of very-high-flow nasal therapy on airway pressure and end-expiratory lung impedance in healthy volunteers. Respir Care. 2015;60(10):1397-1403. doi: $10.4187 /$ respcare. 04028

6. Chanques G, Contantin JM, Sauter M, et al. Discomfort associated with underhumidified high-flow oxygen therapy in critically ill patients. Intensive Care Med. 2009;35(6):996-1003. doi:10.1007/ s00134-009-1538-9

7. Andres D, Thurston N, Brant R, et al. Randomized double-blind trial of the effects of humidified compared with nonhumidified low flow oxygen therapy on the symptoms of patients. Can Respir J. 1997;4:76-80. doi:10.1155/1997/296763

8. Salah B, Dinh Xuan AT, Fouilladieu JL, et al. Nasal mucociliary transport in healthy subjects is slower when breathing dry air. Eur Respir J. 1988;1(9):852-855.

9. Saslow JG, Aghai ZH, Nakhla TA, et al. Work of breathing using high-flow nasal cannula in preterm infants. J Perinatol. 2006;26 (8):476-480. doi:10.1038/sj.jp.7211530

10. Frizzola M, Miller TL, Rodriguez ME, et al. High-flow nasal cannula: impact on oxygenation and ventilation in an acute lung model. Pediatr Pulmonol. 2011;46:67-74. doi:10.1002/ppul.21326 
11. Möller W, Feng S, Domanski U, et al. Nasal high flow reduces dead space. J Appl Physiol (1985). 2017;122(1):191-197.

12. Wettstein RB, Shelledy DC, Peters JI. Delivered oxygen concentrations using low-flow and high-flow nasal cannulas. Respir Care. 2005;50(5):604-609.

13. Groves N, Tobin A. High flow nasal oxygen generates positive airway pressure in adult volunteers. Aust Crit Care. 2007;20(4):126131. doi:10.1016/j.aucc.2007.08.001

14. Ritchie JE, Williams AB, Gerard C. Evaluation of a high flow nasal oxygenation system: gas analysis and pharyngeal pressures. Intensive Care Med. 2006;32:S219.

15. Corley A, Caruana LR, Barnett AG, et al. Oxygen delivery through high-flow nasal cannulae increase end-expiratory lung volume and reduce respiratory rate in post-cardiac surgical patients. Br J Anaesth. 2011;107(6):998-1004. doi:10.1093/bja/aer265

16. Riera J, Perez P, Cortes J, et al. Effect of high-flow nasal cannula and body position on end-expiratory lung volume: a cohort study using electrical impedance tomography. Respir Care. 2013;58(4):589-596. doi: $10.4187 /$ respcare. 02086

17. Plotnikow GA, Thille AW, Vasquez DN, et al. Effects of high-flow nasal cannula on end-expiratory lung impedance in semi-seated healthy subjects. Respir Care. 2018;63(8):1016-1023. doi:10.4187/ respcare. 06116

18. Mündel T, Feng S, Tatkov S, Schneider H. Mechanisms of nasal high flow on ventilation during wakefulness and sleep. J Appl Physiol (1985). 2013;114(8):1058-1065.

19. Okuda M, Tanaka N, Naito K, et al. Evaluation by various methods of the physiological mechanism of a high-flow nasal cannula (HFNC) in healthy volunteers. BMJ Open Respir Res. 2017;4(1):e000200. doi:10.1136/bmjresp-2017-000200

20. Mauri T, Turrini C, Eronia N, et al. Physiologic effects of high-flow nasal cannula in acute hypoxemic respiratory failure. Am J Respir Crit Care Med. 2017;195(9):1207-1215. doi:10.1164/rccm.2017010150WS

21. Bräunlich J, Beyer D, Mai D, et al. Effects of nasal high flow on ventilation in volunteers, COPD and idiopathic pulmonary fibrosis patients. Respiration. 2013;85(4):319-325. doi:10.1159/000342027

22. Sztrymf B, Messika J, Bertrand F, et al. Beneficial effects of humidified high flow nasal oxygen in critical care patients: a prospective pilot study. Intensive Care Med. 2011;37:1780-1786. doi:10.1007/ s00134-011-2180-x

23. Itagaki T, Okuda N, Tsunano $\mathrm{Y}$, et al. Effect of high-flow nasal cannula on thoraco-abdominal synchrony in adult critically ill patients. Respir Care. 2014;59:70-74. doi:10.4187/respcare.02480

24. Pham TM, O'Malley L, Mayfield S, et al. The effect of high flow nasal cannula therapy on the work of breathing in infants with bronchiolitis. Pediatr Pulmonol. 2015;50:713-720. doi:10.1002/ ppul.23060

25. Mauri T, Alban L, Turrini C, et al. Optimum support by high-flow nasal cannula in acute hypoxemic respiratory failure: effects of increasing flow rates. Intensive Care Med. 2017;43(10):1453-1463. doi:10.1007/s00134-017-4860-7

26. Roca O, Riera J, Torres F, et al. High-flow oxygen therapy in acute respiratory failure. Respir Care. 2010;55:408e13.

27. Sztrymf B, Messika J, Mayot T, et al. Impact of high-flow nasal cannula oxygen therapy on intensive care unit patients with acute respiratory failure: a prospective observational study. J Crit Care. 2012;27(3):324.e9-e13. doi:10.1016/j.jcrc.2011.07.075

28. Parke RL, McGuinness SP, Eccleston ML. A preliminary randomized controlled trial to assess effectiveness of nasal high-flow oxygen in intensive care patients. Respir Care. 2011;56:265-270. doi:10.4187/ respcare. 00801

29. Frat JP, Thille AW, Mercat A, et al; for the FLORALI Study Group and the REVA Network High-flow oxygen through nasal cannula in acute hypoxaemic respiratory failure. N Engl J Med. 2015;372:21852196. doi:10.1056/NEJMoa1503326
30. Jones PG, Kamona S, Doran O, Sawtell F, Wilsher M. Randomized controlled trial of humidified high-flow nasal oxygen for acute respiratory distress in the emergency department: the HOT-ER study. Respir Care. 2016;61:291-299. doi:10.4187/respcare.04252

31. Ni YN, Luo J, Yu H, et al. Can high-flow nasal cannula reduce the rate of endotracheal intubation in adult patients with acute respiratory failure with conventional oxygen therapy and noninvasive positive pressure ventilation? A systematic review and meta-analysis. Chest. 2017;151:764-775. doi:10.1016/j.chest.2017.01.004

32. Ni YN, Luo J, Yu H, et al. The effect of high-flow nasal cannula in reducing the mortality and the rate of endotracheal intubation when used before mechanical ventilation compared with conventional oxygen therapy and noninvasive positive pressure ventilation. A systematic review and meta-analysis. Am J Emerg Med. 2018;36 (2):226-233.

33. Lemiale V, Mokart D, Resche-Rigon M, et al; Groupe de Recherche en Réanimation Respiratoire du patient d'Onco-Hématologie (GRRR-OH). Effect of noninvasive ventilation vs oxygen therapy on mortality among immunocompromised patients with acute respiratory failure: a randomized clinical trial. JAMA. 2015;314(16):17111719. doi:10.1001/jama.2015.12402

34. Coudroy R, Jamet A, Petua P, et al. High-flow nasal cannula oxygen therapy versus noninvasive ventilation in immunocompromised patients with acute respiratory failure: an observational cohort study. Ann Intensive Care. 2016;6:45. doi:10.1186/s13613-0160151-7

35. Mokart D, Geay C, Chow-Chine L, et al. High- flow oxygen therapy in cancer patients with acute respiratory failure. Intensive Care Med. 2015;41:2008-2010. doi:10.1007/s00134-015-3994-8

36. Roca O, de Acilu MG, Caralt B, et al. Humidified high flow nasal cannula supportive therapy improves outcomes in lung transplant recipients readmitted to the intensive care unit because of acute respiratory failure. Transplantation. 2015;99:1092-1098. doi:10.1097/TP.0000000000000460

37. Azoulay E, Lemiale V, Mokart D, et al. Effect of high-flow nasal oxygen vs standard oxygen on 28-day mortality in immunocompromised patients with acute respiratory failure: the HIGH randomized clinical trial. JAMA. 2018;320(20):2099-2107. doi:10.1001/ jama.2018.14282

38. Badiger S, John M, Fearnley RA, et al. Optimizing oxygenation and intubation conditions during awake fiberoptic intubation using a highflow nasal oxygendelivery system. Br J Anaesth. 2015;115:629e32. doi:10.1093/bja/aev363

39. Patel A, Nouraei SA. Transnasal Humidified Rapid Insufflation Ventilatory Exchange (THRIVE): a physiological method of increasing apnoea time in patients with difficult airways. Anaesthesia. 2015;70:323e9. doi:10.1111/anae.12923

40. Mir F, Patel A, Iqbal R, et al. A randomised controlled trial comparing transnasal humidified rapid insufflation ventilatory exchange (THRIVE) pre-oxygenation with facemask pre-oxygenation in patients undergoing rapid sequence induction of anaesthesia. Anaesthesia. 2017;72(4):439-443. doi:10.1111/anae.13799

41. Miguel-Montanes R, Haiage D, Messika J, et al. Use of highflow nasal cannula oxygen therapy to prevent desaturation during tracheal intubation of intensive care patients with mild-to-moderate hypoxaemia. Crit Care Med. 2015;43:574e83. doi:10.1097/CCM.0000000000001164

42. Vourc'h M, Asfar P, Volteau C, et al. High-flow nasal cannula oxygen during endotracheal intubation in hypoxaemic patients: a randomized controlled clinical trial. Intensive Care Med. 2015;41:1538.e48-e64. doi:10.1007/s00134-015-3796-z

43. Semler MW, Janz DR, Lentz RJ, FELLOW Investigators, the Pragmatic Critical Care Research Group, et al. Randomized trial of apneic oxygenation during endotracheal intubation of the critically ill. Am J Respir Crit Care Med. 2016;193:273.e80. doi:10.1164/ rccm.201507-1294OC 
44. Baillard C, Fosse JP, Sebbane M, et al. Noninvasive ventilation improves preoxygenation before intubation of hypoxic patients. $\mathrm{Am}$ $J$ Respir Crit Care Med. 2006;174:171-177. doi:10.1164 rccm.200510-1659PP

45. Jaber S, Monnin M, Girard M, et al. Apnoeic oxygenation via highflow nasal cannula oxygen combined with noninvasive ventilation preoxygenation for intubation in hypoxaemic patients in the intensive care unit: the singlecentre, blinded, randomised controlled OPTINIV trial. Intensive Care Med. 2016;42:1877-1887. doi:10.1007/s00134016-4588-9

46. Frat J, Ricard J, Coudroy R On-Behalf-Of REVA Network, et al Preoxygenation with non-invasive ventilation versus high-flow nasal cannula oxygen therapy for intubation of patients with acute hypoxaemic respiratory failure in ICU: the prospective randomised controlled FLORALI-2 study protocol. BMJ Open. 2017;7:e018611.

47. Roca O, Pérez-Terán P, Masclans JR, et al. Patients with New York Heart Association class III heart failure may benefit with high flow nasal cannula supportive therapy: high flow nasal cannula in heart failure. J Crit Care. 2013;28:741-746. doi:10.1016/j.jcrc.2013.02. 007

48. Carratala Perales JM, Llorens P, Brouzet B, et al. High-flow therapy via nasal cannula in acute heart failure. Rev Esp Cardiol. 2011;64:723e5. doi:10.1016/j.recesp.2011.08.002

49. Lucangelo U, Vassallo FG, Marras E, et al. High-flow nasal interface improves oxygenation in patients undergoing bronchoscopy. Crit Care Res Pract. 2012;2012:1-6. doi:10.1155/2012/506382

50. Simon M, Braune S, Frings D, et al. High-flow nasal cannula oxygen versus non-invasive ventilation in patients with acute hypoxaemic respiratory failure undergoing flexible bronchoscopy - a prospective randomised trial. Critical Care. 2014;18(6):712. doi:10.1186/s13054014-0712-9

51. La Combe B, Messika J, Labbé V, et al. High-flow nasal oxygen for bronchoalveolar lavage in acute respiratory failure patients. Eur Respir J. 2016;47:1283-1286. doi:10.1183/13993003.01883-2015

52. Sago T, Harano N, Chogyoji Y, et al. A nasal high-flow system prevents hypoxia in dental patients under intravenous sedation. $J$ Oral Maxillofacial Surg. 2015;73:1058-1064. doi:10.1016/j. joms.2014.12.020

53. Sakazaki R, Suzuki T, Ikeda N. High-flow nasal cannula oxygen supported-transesophageal echocardiography under sedation in a respiratory compromised patient. J Cardiothorac Vasc Anesth. 2018. doi:10.1053/j.jvca.2018.10.009.

54. Schumann R, Natov NS, Rocuts-Martinez KA, et al. High-flow nasal oxygen availability for sedation decreases the use of general anesthesia during endoscopic retrograde cholangiopancreatography and endoscopic ultrasound. World J Gastroenterol. 2016;22:1039810405. doi:10.3748/wjg.v22.i37.8314

55. López-Campos JL, Tan W, Soriano JB. Global burden of COPD. Respirology. 2016;21(1):14-23. doi:10.1111/resp.12660

56. GBD 2015 Chronic Respiratory Disease Collaborators. Global, regional, and national deaths, prevalence, disability-adjusted life years, and years lived with disability for chronic obstructive pulmonary disease and asthma, 1990-2015: a systematic analysis for the Global Burden of Disease Study 2015. Lancet Respir Med. 2017;5 (9):691-706. doi:10.1016/S2213-2600(17)30234-5

57. Ricard JD, Dib F, Esposito-Farese M, et al. Comparison of high flow nasal cannula oxygen and conventional oxygen therapy on ventilatory support duration during acute-on-chronic respiratory failure: study protocol of a multicentre, randomised, controlled trial. The 'HIGHFLOW ACRF' study. BMJ Open. 2018;8(9):e022983. doi:10.1136/ bmjopen-2018-022983

58. Adams CF, Geoghegan PH, Spence CJ, Jermy MC. Modelling nasal high flow therapy effects on upper airway resistance and resistive work of breathing. Respir Physiol Neurobiol. 2018;254:23-29. doi:10.1016/j.resp.2018.03.014
59. Biselli P, Fricke K, Grote L, et al. Reductions in dead space ventilation with nasal high flow depend on physiological dead space volume: metabolic hood measurements during sleep in patients with COPD and controls. Eur Respir J. 2018;51(5):1702251. doi:10.1183/ 13993003.02251-2017

60. Kim ES, Lee H, Kim SJ, et al. Effectiveness of high-flow nasal cannula oxygen therapy for acute respiratory failure with hypercapnia. $J$ Thorac Dis. 2018;10(2):882-888. doi:10.21037/ jtd.2018.01.125

61. Lee MK, Choi J, Park B, et al. High flow nasal cannulae oxygen therapy in acute-moderate hypercapnic respiratory failure. Clin Respir J. 2018;12(6):2046-2056. doi:10.1111/crj.12772

62. Corley A, Rickard CM, Aitken LM, et al. High-flow nasal cannulae for respiratory support in adult intensive care patients. Cochrane Database Syst Rev. 2017;5:CD010172.

63. Doshi P, Whittle JS, Bublewicz M, et al. High-velocity nasal insufflation in the treatment of respiratory failure: a randomized clinical trial. Ann Emerg Med. 2018;72(1):73.e5-83.e5. doi:10.1016/j. annemergmed.2017.12.006

64. Lenglet H, Sztrymf B, Leroy C, et al. Humidified high flow nasal oxygen during respiratory failure in the emergency department: feasibility and efficacy. Respir Care. 2012;57(11):1873-1878. doi:10.4187/respcare.01575

65. Bell N, Hutchinson CL, Green TC, et al. Randomised control trial of humidified high flow nasal cannulae versus standard oxygen in the emergency department. Emerg Med Australas. 2015;27(6):537-541. doi:10.1111/1742-6723.12490

66. Makdee O, Monsomboon A, Surabenjawong U, et al. High-flow nasal cannula versus conventional oxygen therapy in emergency department patients with cardiogenic pulmonary edema: a randomized controlled trial. Ann Emerg Med. 2017;70(4):465.e2-472.e2. doi:10.1016/j.annemergmed.2017.03.028

67. Esquinas AM, Martin C. High-flow nasal cannula oxygen therapy in the emergency department: welcome, but selection should be the first step. Respir Care. 2013;58(5):e66-e67. doi:10.4187/ respcare. 02286

68. Williams AJ. ABC of oxygen: assessing and interpreting arterial blood gases and acid-base balance. BMJ. 1998;317(7167):12131216

69. Kang BJ, Koh Y, Lim C-M, et al. Failure of high-flow nasal cannula therapy may delay intubation and increase mortality. Intensive Care Med. 2015;41:623-632. doi:10.1007/s00134-015-3693-5

70. Esquinas AM, Parke R, Gifford AH. Failure of high-flow nasal cannula and delayed intubation: a new harmful sequence? Intensive Care Med. 2015;41:1170. doi:10.1007/s00134-015-3804-3

71. Ricard J-D, Messika J, Sztrymf B, Gaudry S. Impact on outcome of delayed intubation with high-flow nasal cannula oxygen: is the device solely responsible? Intensive Care Med. 2015;41(6):1157-1158. doi:10.1007/s00134-015-3766-5

72. Roca O, Messika J, Caralt B, et al. Predicting success of high-flow nasal cannula in pneumonia patients with hypoxemic respiratory failure: the utility of the ROX index. J Crit Care. 2016;35:200 205. doi:10.1016/j.jcrc.2016.05.022

73. Roca O, Caralt B, Messika J, et al. An index combining respiratory rate and oxygenation to predict outcome of nasal high flow therapy. Am J Respir Crit Care Med. 2018. doi:10.1164/rccm.2018030589OC

74. Frat JP, Ragot S, Coudroy R, et al. REVA network. Predictors of intubation in patients with acute hypoxemic respiratory failure treated with a noninvasive oxygenation strategy. Crit Care Med. 2018;46 (2):208-215. doi:10.1097/CCM.0000000000002818

75. Koga Y, Kaneda K, Mizuguchi I, et al. Extent of pleural effusion on chest radiograph is associated with failure of high-flow nasal cannula oxygen therapy. J Crit Care. 2016;32:165-169. doi:10.1016/j. jerc. 2015.12.001 
76. Mauri T, Galazzi A, Binda F, et al. Impact of flow and temperature on patient comfort during respiratory support by high-flow nasal cannula. Crit Care. 2018;22:120. doi:10.1186/s13054-017-1926-4

77. Messika J, Ricard JD. Evaluation of risk factors for high flow nasal oxygen failure: a means to avoid disillusion. J Crit Care. 2016;32:222-223. doi:10.1016/j.jcrc.2016.01.014
78. Messika J, Ben Ahmed K, Gaudry S, et al. Use of high-flow nasal cannula oxygen therapy in subjects with ARDS: a 1-year observational study. Respir Care. 2015;60(2):162-169. doi:10.4187/ respcare. 03423

\section{Publish your work in this journal}

The Open Access Emergency Medicine is an international, peerreviewed, open access journal publishing original research, reports, editorials, reviews and commentaries on all aspects of emergency medicine. The manuscript management system is completely online

and includes a very quick and fair peer-review system, which is all easy to use. Visit http://www.dovepress.com/testimonials.php to read real quotes from published authors.

Submit your manuscript here: https://www.dovepress.com/open-access-emergency-medicine-journal 